\title{
The lattice discrepancy of certain three-dimensional bodies
}

\author{
Ekkehard Krätzel and Werner Georg Nowak ${ }^{(*)}$ (Vienna)
}

\begin{abstract}
Based on a very precise approximation to the lattice discrepancy of a Lamé disc, an asymptotic formula is established for the number of lattice points in the three-dimensional body

$$
\left|u_{1}\right|^{m k}+\left(\left|u_{2}\right|^{k}+\left|u_{3}\right|^{k}\right)^{m} \leq x^{m k}
$$

for large real $x$ and fixed reals $m, k$. Particular attention is paid to the boundary points of Gaussian curvature zero.

Mathematics Subject Classification (2000): 11P21, 11N37, 11K38, 52C07.
\end{abstract}

Key words: Lattice points, lattice discrepancy, convex bodies, exponential sums

1. Introduction. For a compact body $\mathcal{B}$ in $\mathbb{R}^{3}$ the lattice point discrepancy of a copy $x \mathcal{B}$, obtained by linear dilation by a large real parameter $x$, is of interest:

$$
P_{\mathcal{B}}(x):=\#\left(x \mathcal{B} \cap \mathbb{Z}^{3}\right)-\operatorname{vol}(\mathcal{B}) x^{3}
$$

The theory of its estimation, resp., asymptotic evaluation has been described in detail in Krätzel's books [13], [15], even in the frame of a more general $s$-dimensional setting, and also in Huxley's monograph [7] which concentrates on the planar case. See also the survey article [11] by the authors with Ivić and Kühleitner.

The case of a convex body $\mathcal{C}$ of smooth boundary $\partial \mathcal{C}$ with bounded nonzero Gaussian curvature throughout is quite well understood. Hlawka's [6] classic bound $P_{\mathcal{C}}(x) \ll x^{3 / 2}$ has been refined up to $O\left(x^{63 / 43+\varepsilon}\right)$ by W. Müller [23], using exponential sums in a most ingenious way. Furthermore, we also know that

$$
P_{\mathcal{C}}(x)=\Omega\left(x(\log x)^{1 / 3}\right), \quad \text { and } \quad \int_{0}^{X}\left(P_{\mathcal{C}}(x)\right)^{2} \mathrm{~d} x \ll X^{3}(\log X)^{2} .
$$

See Nowak [24], and Iosevich, Sawyer \& Seeger [9]. For the special case of the sphere in $\mathbb{R}^{3}$, cf. Heath-Brown [5], for rational ellipsoids Chamizo, Cristóbal \& Ubis [1], for ellipsoids of rotation the present authors [19].

As soon as the boundary $\partial \mathcal{B}$ has points of curvature zero, the problem becomes much harder and, consequently, our knowledge is rather fragmentary. For partial results, see Haberland [4], Krätzel [14], [16], [17], [18] and Peter [26]. A fairly general theorem on bodies of rotation has been established by Nowak [25]. The method used there can

(*) The authors gratefully acknowledge support from the Austrian Science Fund (FWF) under project Nr. P20847-N18. 
be called the "cut-into-slices approach": Since $x \mathcal{B}$ intersects every plane orthogonal to its axis of rotation in a circular disc, a very accurate approximation to the latter's discrepancy is employed, followed by a ("careful") summation with respect to the third coordinate. This idea has also been pursued by the authors for the special case of a rotating Lamé's curve [20].

As a rule, with this sort of problems one cannot expect results which are both very sharp and general. Since the methods used are highly technical, in order to get precise estimates one can only deal with a particular class of bodies at a time. For the expert it will be transparent that the argument developed may be applied in other similar situations as well.

On the basis of this understanding, in the present paper we will concentrate on bodies

$$
\mathcal{B}_{m, k}:=\left\{\left(u_{1}, u_{2}, u_{3}\right) \in \mathbb{R}^{3}:\left|u_{1}\right|^{m k}+\left(\left|u_{2}\right|^{k}+\left|u_{3}\right|^{k}\right)^{m} \leq 1\right\},
$$

where $k>2$ and $m>1$ are fixed real numbers.

Let $A_{m, k}(x)$ the number of integer points in $x \mathcal{B}_{m, k}$, then for integers $k$ and $m$, $A_{m, k}(x)$ describes the average number of representations of integers by the form $\left|u_{1}\right|^{m k}+$ $\left(\left|u_{2}\right|^{k}+\left|u_{3}\right|^{k}\right)^{m}:$

$$
A_{m, k}\left(T^{1 /(m k)}\right)=\sum_{n \leq T} r_{m, k}(n)
$$

where

$$
r_{m, k}(n):=\#\left\{\left(n_{1}, n_{2}, n_{3}\right) \in \mathbb{Z}^{3}:\left|n_{1}\right|^{m k}+\left(\left|n_{2}\right|^{k}+\left|n_{3}\right|^{k}\right)^{m}=n\right\} .
$$

The cases $m=1$ (super sphere) and $k=2$ (rotating Lamé disc) have been dealt with by Krätzel [13], [14], resp., by the authors [20].

An important issue of the present paper is that the cut-into-slices method will be applied for the first time to a body which lacks symmetry of rotation. Thus, the planar discs arising are no longer circular, but they are bounded by Lamé curves. The latter also contain points of curvature zero, which renders the situation much more complicated. We will establish sort of a "truncated Hardy's identity for Lamé discs" which should be of some interest of its own - see Theorem 2 below.

The body $x \mathcal{B}_{m, k}$ has been investigated earlier by Krätzel [18], by a purely "threedimensional" method: This lead to the estimation of double trigonometric sums and to a somewhat less precise final result.

In fact, the Gaussian curvature of $\partial \mathcal{B}_{m, k}$ vanishes on each curve of intersection with one of the coordinate planes. Where any two of these curves meet, there arise flat points, i.e., in $( \pm 1,0,0),(0, \pm 1,0)$, and $(0,0, \pm 1)$. We will be able to evaluate precisely the contribution of these flat points to the lattice discrepancy. The rest of the curves of Gaussian curvature zero will be taken into account by appropriate $O$-terms. (Cf. the remarks after the statement of the main theorem.)

Theorem 1. For fixed reals $k>2, m>1$, with $m k \geq \frac{7}{3}$, and large real $x$, the number $A_{m, k}(x)$ of lattice points in the body $x \mathcal{B}_{m, k}$ satisfies the asymptotic formula

$$
A_{m, k}(x)=\operatorname{vol}\left(\mathcal{B}_{m, k}\right) x^{3}+H_{m k, k, 1}(x)+H_{m k, k, 2}(x)+\mathcal{R}_{m, k}(x),
$$


where

$$
\begin{aligned}
& H_{m k, k, 1}(x)=\mathcal{F}_{m k, k, 1}(x) x^{2-2 / m k}+O(x), \\
& H_{m k, k, 2}(x)=\mathcal{F}_{m k, k, 2}(x) x^{2-1 / m k-1 / k}+O(x),
\end{aligned}
$$

with continuous periodic functions $\mathcal{F}_{m k, k, i}(x), i=1,2$. The functions $H$ and $\mathcal{F}$ will be defined and analyzed in section 2. The remainder term is given by

$$
\begin{aligned}
& \mathcal{R}_{m, k}(x)=O\left(x^{37 / 25}\right)+O\left(x^{\frac{339}{208}-\frac{131}{104 m k}}(\log x)^{\frac{18627 m k-20614}{8320 m k}}\right) \\
& +O\left(x^{\frac{339}{208}-\frac{235}{208 k}}(\log x)^{\frac{18627}{8320}(1-1 / k)}\right) \text {. }
\end{aligned}
$$

Thus,

$$
\mathcal{R}_{m, k}(x) \ll \begin{cases}x^{37 / 25} \quad \text { if } k<\frac{5875}{779}=7.54 \ldots, \text { and } m k<\frac{6550}{779}=8.408 \ldots, \\ x^{\frac{339}{208}-\frac{131}{104 m k}(\log x)^{\frac{18627 m k-20614}{8320 m k}}} \quad \text { if } m k \geq \frac{6550}{779}, \text { and } m \geq \frac{262}{235}=1.11 \ldots, \\ x^{\frac{339}{208}-\frac{235}{208 k}(\log x)^{\frac{18627}{8320}(1-1 / k)}} \quad \text { if } k \geq \frac{5875}{779}, \text { and } m<\frac{262}{235} .\end{cases}
$$

Remarks. As hinted at above, the term $\mathcal{F}_{m k, k, 1}(x) x^{2-2 / m k}$ comes from the points $( \pm 1,0,0)$ where the two Lamé curves

$$
\mathcal{L}_{1}:\left\{\begin{array}{l}
\left|u_{1}\right|^{m k}+\left|u_{2}\right|^{m k}=1, \\
u_{3}=0
\end{array} \quad \text { and } \quad \mathcal{L}_{2}: \quad\left\{\begin{array}{l}
\left|u_{1}\right|^{m k}+\left|u_{3}\right|^{m k}=1, \\
u_{2}=0
\end{array}\right.\right.
$$

intersect. The curvature of either of these curves vanishes at $( \pm 1,0,0)$, of order $m k-2$ (related to the arclength). Similarly, the points $(0, \pm 1,0),(0,0, \pm 1)$ which lie at the intersection of $\mathcal{L}_{1}$, resp., $\mathcal{L}_{2}$ with

$$
\mathcal{L}_{3}:\left\{\begin{array}{l}
\left|u_{2}\right|^{k}+\left|u_{3}\right|^{k}=1 \\
u_{1}=0
\end{array}\right.
$$

contribute the term $\mathcal{F}_{m k, k, 2}(x) x^{2-1 / m k-1 / k}$. The curvature of $\mathcal{L}_{3}$ has zeros of order $k-$ 2 at $(0, \pm 1,0),(0,0, \pm 1)$. As the proof will show, the contribution of the rest of $\mathcal{L}_{1}, \mathcal{L}_{2}$ to the lattice point discrepancy can be bounded by $O\left(x^{\frac{339}{208}-\frac{131}{104 m k}}(\log x)^{\frac{18627 m k-20614}{8320 m k}}\right)$ while $\mathcal{L}_{3}$ without the flat points contributes at most $O\left(x^{\frac{339}{208}-\frac{235}{208 k}}(\log x)^{\frac{18627}{8320}(1-1 / k)}\right)$.

\section{Auxiliary results.}

Lemma 1. (Vaaler's approximation of fractional parts by trigonometric polynomials.) For arbitrary $w \in \mathbb{R}$ and any integer $H>1$, let $\psi(w):=w-[w]-\frac{1}{2}$,

$$
\psi_{H}(w):=-\sum_{0<h<H} \alpha_{h, H} \sin (2 \pi h w), \quad \psi_{H}^{*}(w):=\sum_{0<h<H} \beta_{h, H} \cos (2 \pi h w),
$$


where, for $h=1, \ldots, H-1$,

$$
\alpha_{h, H}:=\frac{1}{\pi h} \rho\left(\frac{h}{H}\right), \quad \beta_{h, H}:=\frac{1}{H}\left(1-\frac{h}{H}\right),
$$

and

$$
\rho(\xi)=\pi \xi(1-\xi) \cot (\pi \xi)+\xi \quad(0<\xi<1) .
$$

Then the following inequality holds true:

$$
\left|\psi(w)-\psi_{H}(w)\right| \leq \psi_{H}^{*}(w)+\frac{1}{2 H} .
$$

Proof. This is one of the main results in Vaaler [27]. A very well readable exposition can also be found in the monograph by Graham and Kolesnik [3].

Lemma 2. (A) Let $F \in C^{4}[A, B]$, and suppose that, for positive parameters $X, Y, Z$, we have $1 \ll B-A \ll X$ and

$$
F^{(j)} \ll X^{2-j} Y^{-1} \quad \text { for } j=2,3,4, \quad\left|F^{\prime \prime}\right| \geq c_{0} Y^{-1},
$$

throughout the interval $[A, B]$, with some constant $c_{0}>0$. Let $\mathcal{J}^{\prime}$ denote the image of $[A, B]$ under $F^{\prime}$, and $F^{*}$ the inverse function of $F^{\prime}$. Set further

$$
r(\xi):= \begin{cases}0 & \text { if } F^{\prime}(\xi) \in \mathbb{Z} \\ \min \left(\left\|F^{\prime}(\xi)\right\|^{-1}, \sqrt{Y}\right) & \text { else }\end{cases}
$$

where $\|\cdot\|$ stands for the distance from the nearest integer. Then, with $e(w)=e^{2 \pi i w}$ as usual,

$$
\begin{aligned}
\sum_{A<n \leq B} e(F(n))= & e\left(\frac{\operatorname{sgn}\left(F^{\prime \prime}\right)}{8}\right) \sum_{\ell \in \mathcal{J}^{\prime}}^{*} \frac{1}{\sqrt{\left|F^{\prime \prime}\left(F^{*}(\ell)\right)\right|}} e\left(F\left(F^{*}(\ell)\right)-\ell F^{*}(\ell)\right)+ \\
& +O(r(A))+O(r(B))+O\left(\log \left(2+\operatorname{length}\left(\mathcal{J}^{\prime}\right)\right)\right)
\end{aligned}
$$

with the notation

$$
\sum_{a \leq n \leq b}^{*} \Phi(n)=\frac{1}{2}\left(\chi_{\mathbb{Z}}(a) \Phi(a)+\chi_{\mathbb{Z}}(b) \Phi(b)\right)+\sum_{a<n<b} \Phi(n),
$$

where $\chi_{\mathbb{Z}}$ is the indicator function of the integers.

(B) Let further $G \in C^{2}[A, B]$, with $G^{(j)} \ll X^{-j} Z$ for $j=0,1,2$, then it follows that

$$
\begin{aligned}
\sum_{A<n \leq B} G(n) e(F(n))= & e\left(\frac{\operatorname{sgn}\left(F^{\prime \prime}\right)}{8}\right) \sum_{\ell \in \mathcal{J}^{\prime}} \frac{G\left(F^{*}(\ell)\right)}{\sqrt{\left|F^{\prime \prime}\left(F^{*}(\ell)\right)\right|}} e\left(F\left(F^{*}(\ell)\right)-\ell F^{*}(\ell)\right)+ \\
& +O\left(Z\left(\sqrt{Y}+\log \left(2+\operatorname{length}\left(\mathcal{J}^{\prime}\right)\right)\right)\right)
\end{aligned}
$$


Proof. Transformation formulas of this kind are quite common, though often with worse error terms. For part (A), see Lemma 2.2 in Kühleitner and Nowak [22]. Part (B) can be found as f. (8.47) in the recent monograph [12] of H. Iwaniec and E. Kowalski.

The functions $H_{a, b, 1}(x)$ and $H_{a, b, 2}(x)$.

For what follows, compare Krätzel [18], and also his monograph [13]. We start with the definition of generalized Bessel functions

$$
J_{\nu}^{(\eta)}(x)=\frac{2}{\sqrt{\pi} \Gamma(\nu+1-1 / \eta)}\left(\frac{x}{2}\right)^{\eta \nu / 2} \int_{0}^{1}\left(1-t^{\eta}\right)^{\nu-1 / \eta} \cos (x t) \mathrm{d} t,
$$

for reals $\eta \geq 1, \nu>1 / \eta$, and real $x>0$. Further, let

$$
\psi_{\nu}^{(\eta)}(x)=2 \sqrt{\pi} \Gamma(\nu+1-1 / \eta) \sum_{n=1}^{\infty}\left(\frac{x}{\pi n}\right)^{\eta \nu / 2} J_{\nu}^{(\eta)}(2 \pi n x),
$$

the series converging absolutely for $\nu>1 / \eta$. On the basis of these functions, we define for reals $a \geq b \geq 2$,

$$
H_{a, b, 1}(x)=\frac{2 \Gamma^{2}(1 / b)}{b \Gamma(2 / b)} \psi_{3 / a}^{(a)}(x),
$$

and

$$
H_{a, b, 2}(x)=8 x \int_{0}^{1} t^{a-1}\left(1-t^{a}\right)^{1 / a-1} \psi_{2 / b}^{(b)}(x t) \mathrm{d} t .
$$

Of course it is important to know the asymptotic behavior of these functions. Since an asymptotics for the generalized Bessel functions is provided by [13, Lemma 3.11], it readily follows that

$$
\begin{aligned}
H_{a, b, 1}(x) & =C_{1}(a, b) x^{2-2 / a} \sum_{n=1}^{\infty} \frac{\sin (2 \pi n x-\pi / a)}{n^{1+2 / a}}+O(x), \\
C_{1}(a, b) & :=\frac{2 \Gamma^{2}(1 / b)}{b \Gamma(2 / b)} \frac{2}{\pi}\left(\frac{a}{2 \pi}\right)^{2 / a} \Gamma\left(1+\frac{2}{a}\right) .
\end{aligned}
$$

To expand $H_{a, b, 2}(x)$, we approximate $J_{2 / b}^{(b)}(2 \pi n x t)$ again by [13, Lemma 3.11], deriving

$$
J_{2 / b}^{(b)}(2 \pi n x t)=\frac{1}{\sqrt{\pi}}\left(\frac{b}{2 \pi n x t}\right)^{1 / b} \sin \left(2 \pi n x t-\frac{\pi}{2 b}\right)+O\left((n x t)^{-1}\right),
$$

at least for $x t$ sufficiently large. The part $\int_{0}^{1 / 2}$ in the definition of $H_{a, b, 2}(x)$ is only an $O(1)$. The arising new integrals

$$
\int_{1 / 2}^{1} t^{a-1 / b}\left(1-t^{a}\right)^{1 / a-1} \sin \left(2 \pi n x t-\frac{\pi}{2 b}\right) \mathrm{d} t
$$


are dealt with according to E.T. Copson [2, p. 24, formula (11.6)]. This finally yields the asymptotics

$$
\begin{aligned}
H_{a, b, 2}(x) & =C_{2}(a, b) x^{2-1 / a-1 / b} \sum_{n=1}^{\infty} n^{-1-1 / a-1 / b} \sin \left(2 \pi n x-\frac{\pi}{2 a}-\frac{\pi}{2 b}\right)+O(x), \\
C_{2}(a, b) & :=\frac{16}{\pi} \frac{a^{1 / a} b^{1 / b}}{(2 \pi)^{1 / a+1 / b}} \Gamma\left(1+\frac{1}{a}\right) \Gamma\left(1+\frac{1}{b}\right) .
\end{aligned}
$$

3. Preparation of the estimate. The "cut-into-slices approach" gives

$$
A_{m, k}(x)=\sum_{\left|n_{1}\right| \leq x} L_{k}\left(\left(x^{m k}-\left|n_{1}\right|^{m k}\right)^{1 / m}\right)
$$

where, for real $W \geq 0$,

$$
L_{k}(W)=\sum_{\left|n_{2}\right|^{k}+\left|n_{3}\right|^{k} \leq W} 1
$$

is the number of lattice points in a Lamé disc with length parameter $W^{1 / k}$. According to $[13$, formulae $(3.57)$ and $(3.47)]$,

$$
L_{k}(W)=a_{k} W^{2 / k}+8 I_{k}(W)-8 \Delta_{k}(W)+O(1),
$$

where $a_{k}=\frac{2 \Gamma^{2}(1 / k)}{k \Gamma(2 / k)}$ is the area of the unit Lamé disc,

$$
\begin{aligned}
I_{k}(W) & :=\int_{0}^{W^{1 / k}} \psi(u) \mathrm{d}\left(\left(W-u^{k}\right)^{1 / k}\right), \\
\Delta_{k}(W) & :=\sum_{\left(\frac{1}{2} W\right)^{1 / k}<n \leq W^{1 / k}} \psi\left(\left(W-n^{k}\right)^{1 / k}\right) .
\end{aligned}
$$

By Euler's formula,

$$
\begin{aligned}
I_{k}(W) & =\frac{1}{2} \sum_{|n|^{k} \leq W}\left(W-|n|^{k}\right)^{1 / k}-\frac{1}{2} \int_{|u|^{k} \leq W}\left(W-|u|^{k}\right)^{1 / k} \mathrm{~d} u \\
& =\frac{1}{2} \sum_{|n|^{k} \leq W}\left(W-|n|^{k}\right)^{1 / k}-\frac{a_{k}}{4} W^{2 / k} .
\end{aligned}
$$

Combining this with (3.1) and (3.2), we obtain

$$
\begin{aligned}
A_{m, k}(x) & =-a_{k} \sum_{\left|n_{1}\right| \leq x}\left(x^{m k}-\left|n_{1}\right|^{m k}\right)^{2 /(m k)}+4 \mathcal{S}(x) \\
& -8 \sum_{\left|n_{1}\right| \leq x} \Delta_{k}\left(\left(x^{m k}-\left|n_{1}\right|^{m k}\right)^{1 / m}\right)+O(x),
\end{aligned}
$$


with

$$
\mathcal{S}(x):=\sum_{\left|n_{2}\right|^{m k}+\left|n_{3}\right|^{m k} \leq x^{m k}}\left(\left(x^{m k}-\left|n_{3}\right|^{m k}\right)^{1 / m}-\left|n_{2}\right|^{k}\right)^{1 / k} .
$$

According to [13, Lemma 3.12],

$$
\begin{aligned}
a_{k} \sum_{\left|n_{1}\right| \leq x}\left(x^{m k}-\left|n_{1}\right|^{m k}\right)^{2 /(m k)} & =\frac{2 \Gamma\left(1+\frac{2}{m k}\right) \Gamma\left(\frac{1}{m k}\right)}{m k \Gamma\left(1+\frac{3}{m k}\right)} a_{k} x^{3}+a_{k} \Psi_{3 /(m k)}^{(m k)}(x) \\
& =\operatorname{vol}\left(\mathcal{B}_{m, k}\right) x^{3}+H_{m k, k, 1}(x)
\end{aligned}
$$

with an appeal to the definition (2.1). Hence we arrive at

$$
\begin{aligned}
A_{m, k}(x) & =-\operatorname{vol}\left(\mathcal{B}_{m, k}\right) x^{3}-H_{m k, k, 1}(x)+4 \mathcal{S}(x) \\
& -8 \sum_{\left|n_{1}\right| \leq x} \Delta_{k}\left(\left(x^{m k}-\left|n_{1}\right|^{m k}\right)^{1 / m}\right)+O(x)
\end{aligned}
$$

This formula already outlines the strategy for the completion of the proof: We will have to evaluate $\mathcal{S}(x)$, and then to estimate the multiple fractional parts sum.

\section{Evaluation of the $\operatorname{sum} \mathcal{S}(x)$.}

Proposition. For large real $x$ and fixed real numbers $m>1$ and $k>2$, the sum $\mathcal{S}(x)$ defined in (3.3) satisfies the asymptotic formula

$$
\mathcal{S}(x)=\frac{1}{2} \operatorname{vol}\left(\mathcal{B}_{m, k}\right) x^{3}+\frac{1}{2} H_{m k, k, 1}(x)+\frac{1}{4} H_{m k, k, 2}(x)+O\left(x^{\frac{339}{208}-\frac{235}{208 k}}(\log x)^{\frac{18627}{8320}(1-1 / k)}\right) .
$$

Proof. Obviously,

$$
\frac{1}{4} \mathcal{S}(x)=\sum_{\substack{n_{2}^{m k}+n_{3}^{m k} \leq x m k \\ n_{2}, n_{3} \geq 0}}^{\prime \prime}\left(\left(x^{m k}-n_{3}^{m k}\right)^{1 / m}-n_{2}^{k}\right)^{1 / k}=\sum_{\substack{\left(t_{1}^{k}+n_{2}^{k}\right)^{m}+n_{3}^{m k} \leq x m k \\ t_{1}, n_{2}, n_{3} \geq 0}}^{\prime \prime} \int_{\substack{m \\ n^{\prime}}} \mathrm{d} t_{1}
$$

where $\sum^{\prime \prime}$ means that terms corresponding to $n_{2} n_{3}=0$ get weight $\frac{1}{2}$, and the term with $n_{2}=n_{3}=0$ gets weight $\frac{1}{4}$. We subdivide this sum into 6 subsums, according to the relative size of the three variables of summation, resp., integration, $t_{1}, n_{2}, n_{3}$. Thus $\mathcal{S}_{1,2,3}(x)$ comprehends the case $t_{1} \leq n_{2} \leq n_{3}$, and so on. Terms with $n_{2}=n_{3}$ get weight $\frac{1}{2}$, which is symbolized by the notation $\sum^{\prime}$. Thus,

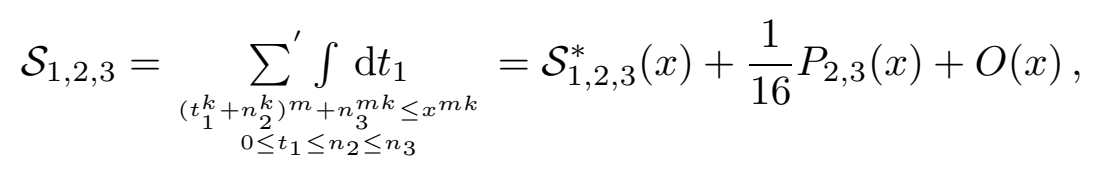


where

$$
\mathcal{S}_{1,2,3}^{*}(x)=\sum_{\substack{\left(t_{1}^{k}+t_{2}^{k}\right)^{m}+n_{3}^{m k} \leq x^{m k} \\ 0 \leq t_{1} \leq t_{2} \leq n_{3}}} \mathrm{~d}\left(t_{1}, t_{2}\right)
$$

and $P_{2,3}(x)$ has been defined in $[18$, formula $(9)]$, with $(k, \kappa)$ instead of $(m k, k)$. It satisfies the estimate

$$
P_{2,3}(x) \ll x^{\frac{339}{208}-\frac{235}{208 k}}(\log x)^{(18627 / 8320)(1-1 / k)},
$$

according to the first formula on top of page 768 of [18], if one replaces M. Huxley's bound in [7] by his more recent slight improvement in [8]. Further, by the Euler summation formula,

$$
\begin{aligned}
& \mathcal{S}_{1,2,3}^{*}(x)=\quad \iiint \mathrm{d}\left(t_{1}, t_{2}, t_{3}\right)- \\
& \begin{array}{c}
\left(t_{1}^{k}+t_{2}^{k}\right)^{m}+t_{3}^{m k} \leq x^{m k} \\
0 \leq t_{1} \leq t_{2} \leq t_{3}
\end{array} \\
& \text { - } \quad \iint \psi\left(\left(x^{m k}-\left(t_{1}^{k}+t_{2}^{k}\right)^{m}\right)^{1 /(m k)}\right) \mathrm{d}\left(t_{1}, t_{2}\right)+O(x) \\
& 0 \leq t_{1}^{m k} \leq t_{2}^{m k} \leq x^{m k}-\left(t_{1}^{k}+t_{2}^{k}\right)^{m} \\
& =\quad \iiint \mathrm{d}\left(t_{1}, t_{2}, t_{3}\right)+\frac{1}{16} H_{m k, k, 1}(x)+O(x), \\
& \begin{array}{c}
\left(t_{1}^{k}+t_{2}^{k}\right)^{m}+t_{3}^{m k} \leq x^{m k} \\
0 \leq t_{1} \leq t_{2} \leq t_{3}
\end{array}
\end{aligned}
$$

by $[18$, formula (12)] and the formulae at the bottom of p. 762 of [18]. Thus, altogether,

$$
\mathcal{S}_{1,2,3}(x)=\iiint_{\substack{\left(t_{1}^{k}+t_{2}^{k}\right)^{m}+t_{3}^{m k} \leq x m k \\ 0 \leq t_{1} \leq t_{2} \leq t_{3}}} \mathrm{~d}\left(t_{1}, t_{2}, t_{3}\right)+\frac{1}{16} H_{m k, k, 1}(x)+O\left(x^{\frac{339}{208}-\frac{235}{208 k}}(\log x)^{\frac{18627}{8320}(1-1 / k)}\right) .
$$

Similarly,

$$
\mathcal{S}_{1,3,2}(x)=\mathcal{S}_{1,3,2}^{*}(x)+\frac{1}{16} P_{3,2}(x)+O(x),
$$

where

$$
\mathcal{S}_{1,3,2}^{*}(x)=\sum_{\substack{\left(t_{1}^{k}+n_{2}^{k}\right)^{m}+t_{3}^{m k} \leq x^{m k} \\ 0 \leq t_{1} \leq t_{3} \leq n_{2}}} \int_{\substack{m \\ \text { a }}} \mathrm{d}\left(t_{1}, t_{3}\right)
$$

and $P_{3,2}(x)$ has been defined in [18, formula (16)], with $(k, \kappa)$ instead of $(m k, k)$. It satisfies the same estimate as $P_{2,3}(x)$, appealing to [18, Lemma 2]. Consequently, again by Euler's formula,

$$
\mathcal{S}_{1,3,2}(x)=\iiint_{\substack{\left(t_{1}^{k}+t_{2}^{k}\right)^{m}+t_{3}^{m k} \leq x m k \\ 0 \leq t_{1} \leq t_{3} \leq t_{2}}} \mathrm{~d}\left(t_{1}, t_{2}, t_{3}\right)+H_{3,2}^{*}(x)+O\left(x^{\frac{339}{208}-\frac{235}{208 k}}(\log x)^{\frac{18627}{8320}(1-1 / k)}\right)
$$


with

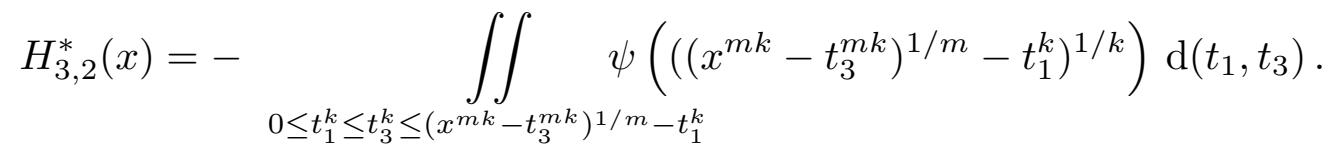

Moreover,

$$
\mathcal{S}_{2,1,3}(x)=\mathcal{S}_{2,1,3}^{*}(x)+P_{1,3}^{*}(x)+O(x),
$$

where

$$
\mathcal{S}_{2,1,3}^{*}(x)=\sum_{\substack{\left(t_{1}^{k}+t_{2}^{k}\right)^{m}+n_{3}^{m k} \leq x^{m k} \\ 0 \leq t_{2} \leq t_{1} \leq n_{3}}} \mathrm{~d}\left(t_{1}, t_{2}\right)
$$

and

$$
\left.P_{1,3}^{*}(x)=-\sum_{2^{-m}\left(x^{m k}-n_{3}^{m k}\right) \leq t_{1}^{m k} \leq x^{m k}-n_{3}^{m k}} \psi d\left(\left(x^{m k}-n_{3}^{m k}\right)^{1 / m}-t_{1}^{k}\right)^{1 / k}\right) .
$$

The substitution

$$
z=\left(\left(x^{m k}-n_{3}^{m k}\right)^{1 / m}-t_{1}^{k}\right)^{1 / k} \Leftrightarrow t_{1}=\left(\left(x^{m k}-n_{3}^{m k}\right)^{1 / m}-z^{k}\right)^{1 / k}
$$

yields

$$
P_{1,3}^{*}(x)=\sum_{0 \leq z^{m k} \leq 2^{-m}\left(x^{m k}-n_{3}^{m k}\right)} \psi(z) z^{k-1}\left(\left(x^{m k}-n_{3}^{m k}\right)^{1 / m}-z^{k}\right)^{-1+1 / k} .
$$

Hence, integrating by parts, we conclude that

$$
P_{1,3}^{*}(x) \ll x .
$$

Furthermore, essentially repeating an earlier argument,

$$
\begin{aligned}
& \mathcal{S}_{2,1,3}^{*}(x)=\quad \iiint_{\substack{\left.t^{k}+t^{k}\right)^{m}+t_{3}^{m k} \leq x^{m k} \\
0 \leq t_{2} \leq t_{1} \leq t_{3}}} \mathrm{~d}\left(t_{1}, t_{2}, t_{3}\right)- \\
& -\quad \iint \psi\left(\left(x^{m k}-\left(t_{1}^{k}+t_{2}^{k}\right)^{m}\right)^{1 /(m k)}\right) \mathrm{d}\left(t_{1}, t_{2}\right)+O(x) \\
& 0 \leq t_{2}^{m k} \leq t_{1}^{m k} \leq x^{m k}-\left(t_{1}^{k}+t_{2}^{k}\right)^{m} \\
& =\quad \iiint \mathrm{d}\left(t_{1}, t_{2}, t_{3}\right)+\frac{1}{16} H_{m k, k, 1}(x)+O(x) . \\
& \begin{array}{c}
\left(t_{1}^{k}+t_{2}^{k}\right)^{m}+t_{3}^{m k} \leq x^{m k} \\
0 \leq t_{2} \leq t_{1} \leq t_{3}
\end{array}
\end{aligned}
$$

Hence also

$$
\mathcal{S}_{2,1,3}^{*}(x)=\quad \iiint_{\substack{\left(t_{1}^{k}+t_{2}^{k}\right)^{m}+t_{3}^{m k} \leq x m k \\ 0 \leq t_{2} \leq t_{1} \leq t_{3}}} \mathrm{~d}\left(t_{1}, t_{2}, t_{3}\right)+\frac{1}{16} H_{m k, k, 1}(x)+O(x) .
$$


Next,

$$
\mathcal{S}_{2,3,1}(x)=\mathcal{S}_{2,3,1}^{*}(x)+P_{3,1}^{*}(x)+O(x),
$$

with

$$
\mathcal{S}_{2,3,1}^{*}(x)=\sum_{\substack{\left(t_{1}^{k}+t_{2}^{k}\right)^{m}+n_{3}^{m k} \leq x^{m k} \\ 0 \leq t_{2} \leq n_{3} \leq t_{1}}} \mathrm{~d}\left(t_{1}, t_{2}\right)
$$

and

$$
P_{3,1}^{*}(x)=-\sum_{\left(x^{m k}-n_{3}^{m k}\right)^{1 / m}-n_{3}^{k} \leq t_{1}^{k} \leq\left(x^{m k}-n_{3}^{m k}\right)^{1 / m}} \mathrm{~d}_{1} \psi\left(\left(\left(x^{m k}-n_{3}^{m k}\right)^{1 / m}-t_{1}^{k}\right)^{1 / k}\right) .
$$

Again by [18, Lemma 1], we bound $P_{3,1}^{*}(x)$ by $O\left(x^{\frac{339}{208}-\frac{235}{208 k}}(\log x)^{\frac{18627}{8320}(1-1 / k)}\right)$ as well. Further,

$$
\mathcal{S}_{2,3,1}^{*}(x)=\quad \iiint_{\substack{\left.t_{1}^{k}+t_{2}^{k}\right) m \\ 0 \leq t_{2} \leq t_{3} \leq t_{1} \\ 0 \leq t_{1}}} \mathrm{~d}\left(t_{1}, t_{2}, t_{3}\right)+H_{3,1}^{*}(x)+O(x)
$$

where

$$
\begin{aligned}
& H_{3,1}^{*}(x)=-\underset{0 \leq t_{2}^{m k} \leq x^{m k}-\left(t_{1}^{k}+t_{2}^{k}\right)^{m} \leq t_{1}^{m k}}{\int} \psi\left(\left(x^{m k}-\left(t_{1}^{k}+t_{2}^{k}\right)^{m}\right)^{1 /(m k)}\right) \mathrm{d}\left(t_{1}, t_{2}\right) \\
& =\iint(z) z^{m k-1}\left(x^{m k}-z^{m k}\right)^{-1+1 / m}\left(\left(x^{m k}-z^{m k}\right)^{1 / m}-t_{2}^{k}\right)^{-1+1 / k} \mathrm{~d}\left(z, t_{2}\right), \\
& 0 \leq t_{2}^{k} \leq z^{k} \leq\left(x^{m k}-z^{m k}\right)^{1 / m}-t_{2}^{k}
\end{aligned}
$$

on the basis of the substitution

$$
z=\left(x^{m k}-\left(t_{1}^{k}+t_{2}^{k}\right)^{m}\right)^{1 /(m k)} \Leftrightarrow t_{1}=\left(\left(x^{m k}-z^{m k}\right)^{1 / m}-t_{2}^{k}\right)^{1 / k} .
$$

Observing that $t_{2}^{k} \leq \frac{1}{2}\left(x^{m k}-z^{m k}\right)^{1 / m}$ and $z^{m k} \leq \frac{1}{2} x^{m k}$, an integration by parts shows that $H_{3,1}^{*}(x) \ll x$. Hence, altogether,

$$
\mathcal{S}_{2,3,1}(x)=\quad \iiint_{\substack{\left(t_{1}^{k}+t_{2}^{k}\right) m \\ 0 \leq t_{2} \leq t_{3} \leq t_{1}}} \mathrm{~d}\left(t_{1}, t_{2}, t_{3}\right) \quad+O\left(x^{\frac{339}{208}-\frac{235}{208 k}}(\log x)^{\frac{18627}{8320}(1-1 / k)}\right)
$$

Further on,

$$
\mathcal{S}_{3,1,2}(x)=\mathcal{S}_{3,1,2}^{*}(x)+P_{1,2}^{*}(x)+O(x)
$$

with




and

$$
P_{1,2}^{*}(x)=-\sum_{x^{m k}-t_{1}^{m k} \leq\left(t_{1}^{k}+n_{2}^{k}\right)^{m} \leq x^{m k}} \int_{\substack{m \\ 0 \leq t_{1} \leq n_{2}}} \mathrm{~d} t_{1} \psi\left(\left(x^{m k}-\left(t_{1}^{k}+n_{2}^{k}\right)^{m}\right)^{1 /(m k)}\right) .
$$

In order to bound $P_{1,2}^{*}(x)$, we substitute

$$
z=\left(x^{m k}-\left(t_{1}^{k}+n_{2}^{k}\right)^{m}\right)^{1 /(m k)} \quad \Leftrightarrow \quad t_{1}=\left(\left(x^{m k}-z^{m k}\right)^{1 / m}-n_{2}^{k}\right)^{1 / k}
$$

and obtain

$$
P_{1,2}^{*}(x)=\underset{\left(z^{k}+n_{2}^{k}\right)^{m} \leq x^{m k}-z^{m k} \leq 2^{m} n_{2}^{m k}, z \geq 0}{\sum_{n_{2}} \mathrm{~d} z} \psi(z)
$$

where

$$
\varphi_{n_{2}}(z)=z^{m k-1}\left(x^{m k}-z^{m k}\right)^{-1+1 / m}\left(\left(x^{m k}-z^{m k}\right)^{1 / m}-n_{2}^{k}\right)^{-1+1 / k}
$$

The condition $\left(z^{k}+n_{2}^{k}\right)^{m} \leq x^{m k}-z^{m k}$ on the one hand implies that $z^{m k} \leq \frac{1}{2} x^{m k}$, hence $\left(x^{m k}-z^{m k}\right)^{-1+1 / m} \asymp x^{k-m k}$. On the other hand, $\left(x^{m k}-z^{m k}\right)^{1 / m} \geq z^{k}+n_{2}^{k}$, thus $\left(\left(x^{m k}-z^{m k}\right)^{1 / m}-n_{2}^{k}\right)^{-1+1 / k} \leq z^{1-k}$. Therefore, altogether,

$$
\varphi_{n_{2}}(z) \ll x^{k-m k} z^{m k-k} \ll 1
$$

uniformly in $n_{2}$. Since $\varphi_{n_{2}}(z)$ increases monotonically with $z$, integration by parts readily gives

$$
P_{1,2}^{*}(x) \ll x
$$

Further,

$$
\mathcal{S}_{3,1,2}^{*}(x)=\quad \iiint_{\substack{\left(t_{1}^{k}+t_{2}^{k}\right)^{m}+t_{3}^{m k} \leq x^{m k} \\ 0 \leq t_{3} \leq t_{1} \leq t_{2}}} \mathrm{~d}\left(t_{1}, t_{2}, t_{3}\right)+H_{1,2}^{*}(x)+O(x),
$$

where

$$
\left.H_{1,2}^{*}(x)=-\underset{0 \leq t_{3}^{m k} \leq t_{1}^{m k} \leq 2^{-m}\left(x^{m k}-t_{3}^{m k}\right)}{\iint_{1}} \psi\left(\left(x^{m k}-t_{3}^{m k}\right)^{1 / m}-t_{1}^{k}\right)^{1 / k}\right) \mathrm{d}\left(t_{1}, t_{3}\right) .
$$

Collecting results, we arrive at

$$
\mathcal{S}_{3,1,2}(x)=\quad \iiint_{\substack{\left(t_{1}^{k}+t_{2}^{k}\right) m_{+t_{3}^{m} \leq x m k} \\ 0 \leq t_{3} \leq t_{1} \leq t_{2}}} \mathrm{~d}\left(t_{1}, t_{2}, t_{3}\right)+H_{1,2}^{*}(x)+O(x) .
$$

Finally,

$$
\mathcal{S}_{3,2,1}(x)=\mathcal{S}_{3,2,1}^{*}(x)+P_{2,1}^{*}(x)+O(x),
$$


with

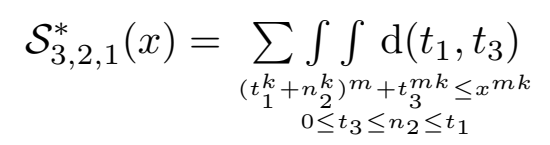

and

$$
P_{2,1}^{*}(x)=-\sum_{x^{m k}-n_{2}^{m k} \leq\left(t_{1}^{k}+n_{2}^{k}\right)^{m} \leq x^{m k}} \underset{\substack{m n_{2} \leq t_{1} \\ 0 .}}{ } \mathrm{d} t_{1} \psi\left(\left(x^{m k}-\left(t_{1}^{k}+n_{2}^{k}\right)^{m}\right)^{1 /(m k)}\right) .
$$

Quite the same analysis as used before for $P_{1,2}^{*}(x)$ applies again and yields

$$
P_{2,1}^{*}(x) \ll x
$$

Furthermore,

$$
\mathcal{S}_{3,2,1}^{*}(x)=\quad \iiint_{\substack{\left(t_{1}^{k}+t_{2}^{k}\right)^{m}+t_{3}^{m k} \leq x^{m k} \\ 0 \leq t_{3} \leq t_{2} \leq t_{1}}} \mathrm{~d}\left(t_{1}, t_{2}, t_{3}\right)+H_{2,1}^{*}(x)+O(x)
$$

where

$$
\left.H_{2,1}^{*}(x)=-\underset{0 \leq t_{3}^{k} \leq\left(x^{m k}-t_{3}^{m k}\right)^{1 / m}-t_{1}^{k} \leq t_{1}^{k}}{\iint_{1}} \psi\left(\left(x^{m k}-t_{3}^{m k}\right)^{1 / m}-t_{1}^{k}\right)^{1 / k}\right) \mathrm{d}\left(t_{1}, t_{3}\right) .
$$

We transform this double integral by the substitution

$$
z=\left(\left(x^{m k}-t_{3}^{m k}\right)^{1 / m}-t_{1}^{k}\right)^{1 / k} \Leftrightarrow t_{1}=\left(\left(x^{m k}-t_{3}^{m k}\right)^{1 / m}-z^{k}\right)^{1 / k}
$$

to obtain

$$
H_{2,1}^{*}(x)=\underset{0 \leq t_{3}^{k} \leq z^{k} \leq \frac{1}{2}\left(x^{m k}-t_{3}^{m k}\right)^{1 / m}}{\iint_{i}} \psi(z) z^{k-1}\left(\left(x^{m k}-t_{3}^{m k}\right)^{1 / m}-z^{k}\right)^{-1+1 / k} \mathrm{~d}\left(t_{3}, z\right) .
$$

Integration by parts shows again that $H_{2,1}^{*}(x) \ll x$, hence

$$
\mathcal{S}_{3,2,1}(x)=\quad \iiint_{\substack{\left(t_{1}^{k}+t_{2}^{k}\right)^{m}+t_{3}^{m k} \leq x \\ 0 \leq t_{3} \leq t_{2} \leq t_{1}}} \mathrm{~d}\left(t_{1}, t_{2}, t_{3}\right)+O(x) .
$$

Adding up the results (4.1) - (4.6), we get

$$
\begin{aligned}
\frac{1}{4} \mathcal{S}(x) & =\frac{1}{8} \operatorname{vol}\left(\mathcal{B}_{m, k}\right) x^{3}+\frac{1}{8} H_{m k, k, 1}(x)+H_{3,2}^{*}(x)+H_{1,2}^{*}(x) \\
& +O\left(x^{\frac{339}{208}-\frac{235}{208 k}}(\log x)^{\frac{18627}{8320}(1-1 / k)}\right) .
\end{aligned}
$$


Finally,

$$
H_{3,2}^{*}(x)+H_{1,2}^{*}(x)=-\iint_{\max \left(2^{m} t_{1}^{m k},\left(t_{1}^{k}+t_{3}^{k}\right)^{m}\right) \leq x^{m k}-t_{3}^{m k}, t_{1}, t_{3} \geq 0} \psi\left(\left(\left(x^{m k}-t_{3}^{m k}\right)^{1 / m}-t_{1}^{k}\right)^{1 / k}\right) \mathrm{d}\left(t_{1}, t_{3}\right) .
$$

According to [18], formulae (15) and (20), and p. 763-764, this last double integral equals $\frac{1}{16} H_{m k, k, 2}(x)$. This observation completes the proof of the Proposition.

We conclude this section by the remark that using the Proposition in (3.6) gives

$$
\begin{aligned}
A_{m, k}(x) & =\operatorname{vol}\left(\mathcal{B}_{m, k}\right) x^{3}+H_{m k, k, 1}(x)+H_{m k, k, 2}(x) \\
& -8 \sum_{\left|n_{1}\right| \leq x} \Delta_{k}\left(\left(x^{m k}-\left|n_{1}\right|^{m k}\right)^{1 / m}\right)+O\left(x^{\frac{339}{208}-\frac{235}{208 k}}(\log x)^{\frac{18627}{8320}(1-1 / k)}\right) .
\end{aligned}
$$

5. Approximating the lattice discrepancy of the Lamé disc. The lattice point discrepancy of the disc $\left|u_{1}\right|^{k}+\left|u_{2}\right|^{k} \leq W$ is expressed by (3.2). In fact, for $I_{k}(W)$ a very precise evaluation is known, namely

$$
I_{k}(W)=\frac{1}{\pi}\left(\frac{k}{2 \pi}\right)^{1 / k} \Gamma\left(1+\frac{1}{k}\right) W^{1 / k-1 / k^{2}} \sum_{j=1}^{\infty} j^{-1-1 / k} \sin \left(2 \pi j W^{1 / k}-\frac{\pi}{2 k}\right)+O(1) .
$$

See [13, p. 148]. For $\Delta_{k}(W)$ as defined in (3.3), the sharpest upper bound known reads

$$
\Delta_{k}(W)=O\left(W^{\frac{131}{208 k}}(\log W)^{\frac{18627}{8320}}\right) .
$$

This follows by the argument of Kuba [21], if one uses Huxley's method in its most recent form [8]. Our present target will be to approximate $\Delta_{k}(W)$ by trigonometric polynomials, with a fairly small $O$-term. This result is intended to be useful for further calculations involving $\Delta_{k}(W)$, like a summation with respect to a third dimension, in the sense of (3.1).

To this end, recall the notation of Lemma 1 and let $H_{W}$ denote an arbitrary map from $\left\{n \in \mathbb{Z}^{+}: \frac{1}{2} W<n^{k} \leq W\right\}$ into the integers exceeding 1 , so that $\max \left(\log H_{W}\right) \ll$ $\log W$. Further, define $\Delta_{k, H_{W}}(W)$ and $\Delta_{k, H_{W}}^{*}(W)$ analogously to $\Delta_{k}(W)$, replacing $\psi$ by $\psi_{H_{W}(n)}$, resp., $\psi_{H_{W}(n)}^{*}$, for every value of $n$ in (5.1). By Lemma 1,

$$
\left|\Delta_{k}(W)-\Delta_{k, H}(W)\right| \leq \Delta_{k, H}^{*}(W)+\sum_{\left(\frac{1}{2} W\right)^{1 / k}<n \leq W^{1 / k}} \frac{1}{H_{W}(n)} .
$$

Put for short $\alpha=\left(\alpha_{h, H_{W}(n)}\right)_{0<h<H_{W}(n)}, \beta=\left(\beta_{h, H_{W}(n)}\right)_{0<h<H_{W}(n)}$, and write $\gamma=$ $\left(\gamma_{h, H_{W}(n)}\right)_{0<h<H_{W}(n)}$ for either $\alpha$ or $\beta$. In fact, $\Delta_{k, H_{W}(n)}(W)$ and $\Delta_{k, H_{W}(n)}^{*}(W)$ can be transformed by the same calculation, considering exponential sums

$$
E_{\gamma}:=\sum_{\left(\frac{1}{2} W\right)^{1 / k}<n \leq W^{1 / k}} \sum_{0<h<H_{W}(n)} \gamma_{h, H_{W}(n)} e\left(-h\left(W-n^{k}\right)^{1 / k}\right) .
$$


We divide the range of $n$ by a dyadic sequence $N_{0}<N_{1}<\ldots<N_{J}$ defined by the condition

$$
\left.\frac{d}{d u}\left(-h\left(W-u^{k}\right)^{1 / k}\right)\right|_{u=N_{j}}=h 2^{j} \quad \Longleftrightarrow \quad N_{j}=W^{1 / k}\left(1+2^{-j q}\right)^{-1 / k}
$$

with

$$
q:=\frac{k}{k-1}
$$

for short, throughout what follows. $J$ is chosen as

$$
J:=\left[\frac{\log \left(\left(W / c_{0}\right)^{(1-\lambda) / k}\right)}{q \log 2}\right]+1,
$$

where $c_{0} \in\left[1, b_{0}\right]$ is any real, and $0 \leq \lambda<1, b_{0} \geq 1$ are arbitrary constants. Hence $J \ll \log W$, and $W^{1 / k}-N_{J} \asymp W^{\lambda / \bar{k}}$. We further impose the condition that $H_{W}$ is constant on each subinterval $] N_{j}, N_{j+1}$ ], say equal to $H_{W, j}$, for $j=0,1, \ldots, J-1$. By simple calculations,

$$
N_{j+1}-N_{j} \asymp 2^{-j q} W^{1 / k}
$$

and

$$
\frac{d^{2}}{d u^{2}}\left(-h\left(W-u^{k}\right)^{1 / k}\right)=(k-1) h W u^{k-2}\left(W-u^{k}\right)^{1 / k-2} \asymp h 2^{j q(1 / k-2)} W^{-1 / k}
$$

for $u \in\left[N_{j}, N_{j+1}\right]$. Thus we may apply Lemma 2, part (A), to each of the subintervals $\left[N_{j}, N_{j+1}\right]$, with the choice of parameters $X=2^{-j q} W^{1 / k}, Y=h^{-1} 2^{-j q(1 / k-2)} W^{1 / k}$. This yields

$$
\begin{gathered}
\sum_{0<h<H_{W, j}} \gamma_{h, H_{W, j}} \sum_{N_{j}<n \leq N_{j+1}} e\left(-h\left(W-n^{k}\right)^{1 / k}\right)= \\
=\frac{W^{1 /(2 k)}}{\sqrt{k-1}} \sum_{0<h<H_{W, j}} \gamma_{h, H_{W, j}} h \sum_{\ell=2^{j} h}^{2^{j+1} h} \frac{(h \ell)^{q / 2-1}}{\left(h^{q}+\ell^{q}\right)^{1-1 /(2 q)}} e\left(-W^{1 / k}\left(h^{q}+\ell^{q}\right)^{1 / q}+\frac{1}{8}\right) \\
+O\left((\log W)^{2}\right)
\end{gathered}
$$

since, by the definitions in Lemma $1, \gamma_{h, H_{W, j}} \ll h^{-1}$. Using the real and imaginary parts and taking into account the appropriate portion of (5.2), we readily infer the following result.

Theorem 2. Let $W$ be a large parameter, $k>2$ and $0 \leq \lambda<1$ fixed real numbers, $H_{W, j}>1$ integers, $j=0,1, \ldots, J-1$, as explained above, with $\max _{j} \log H_{W, j} \ll \log W$, and $J$ given by (5.5). For $\alpha$ and $\beta$ as defined, and $\gamma$ denoting either $\alpha$ or $\beta$, put

$$
\begin{aligned}
\sum_{H, W, k, j}^{(\gamma)}:=\frac{W^{1 /(2 k)}}{\sqrt{k-1}} \sum_{0<h<H_{W, j}} \gamma_{h, H_{W, j}} h \sum_{\ell=2^{j} h}^{2^{j+1} h}(h \ell)^{q / 2-1}\left(h^{q}+\ell^{q}\right)^{-1+1 /(2 q)} \times \\
\times e\left(W^{1 / k}\left(h^{q}+\ell^{q}\right)^{1 / q}-\frac{1}{8}\right) .
\end{aligned}
$$


Then the remainder term $\Delta_{k}(W)$ defined in (3.3) for the number of lattice points in a Lamé disc satisfies the estimate

$$
\begin{aligned}
& \left|\Delta_{k}(W)-\sum_{j=0}^{J-1} \Im\left(\sum_{H, W, k, j}^{(\alpha)}\right)\right| \\
& \leq \sum_{j=0}^{J-1} \Re\left(\sum_{H, W, k, j}^{(\beta)}\right)+\sum_{j=0}^{J-1} \frac{\left.\left.\#(] N_{j}, N_{j+1}\right] \cap \mathbb{Z}\right)}{H_{W, j}}+C\left(W^{\lambda / k}+(\log W)^{3}\right),
\end{aligned}
$$

with an appropriate constant $C>0$.

Remark. This result may be called a "truncated Hardy's identity for Lamé discs". It may well be compared with its classic counterpart for the circle, namely

$$
\sum_{0 \leq n \leq X} r(n)-\pi X=\frac{1}{\pi} X^{1 / 4} \sum_{1 \leq n \leq Y} \frac{r(n)}{n^{3 / 4}} \cos (2 \pi \sqrt{n X}-3 \pi / 4)+O\left(X^{1 / 2+\varepsilon} Y^{-1 / 2}\right)+O\left(Y^{\varepsilon}\right)
$$

where $X, Y$ are any large reals: see Ivić [10, f. (1.9)]. Obviously, the formula in Theorem 2 is much more complicated. Apart from the technical smoothing factors $\alpha$ and $\beta$, this is due to the fact that a Lamé curve has points of curvature zero, and therefore unavoidable.

6. Estimation of the multiple exponential sum. Going back to (4.7), it remains to bound

$$
\sum_{\left|n_{1}\right| \leq x} \Delta_{k}\left(\left(x^{m k}-\left|n_{1}\right|^{m k}\right)^{1 / m}\right)
$$

Let $U$ be a further parameter to be chosen later in terms of $x$. For $\left|n_{1}\right| \leq U$, we use $(5.1)$, to get a total error of $O\left(U x^{\frac{131}{208}}(\log x)^{\frac{18627}{8320}}\right)$. The remaining range $U<n_{1} \leq x$ will be divided into dyadic subintervals in various ways: First, we apply Theorem 2, with $\lambda=0.47$, to approximate $\Delta_{k}\left(\left(x^{m k}-\left|n_{1}\right|^{m k}\right)^{1 / m}\right)$. This gives an overall error term of $O\left(x^{1.47}\right)$. Next, assuming w.l.o.g. that $\frac{x}{U}$ is a power of 2 , we define $\left.\left.\mathcal{U}_{r}:=\right] 2^{r-1} U, 2^{r} U\right], r=1, \ldots, R_{1}$, where $2^{R_{1}} U=\frac{x}{2}$, hence $R_{1} \ll \log x$. Further, $\left.\left.\mathcal{V}_{r}:=\right] x\left(1-2^{-r}\right), x\left(1-2^{-r-1}\right)\right], r=1, \ldots, R_{2}$, with $R_{2}$ such that $2^{-R_{2}-1} x<10$, say. Obviously, also $R_{2} \ll \log x$, and the remaining range gives only a small error of $O(x)$. After these preparations, and appealing to Prop. 2, it will obviously suffice to bound all sums

$$
\left.\sum_{n_{1} \in \mathcal{I}} \sum_{H, W, k, j}^{(\gamma)}\right|_{W=\left(x^{m k}-n_{1}^{m k}\right)^{1 / m}}
$$

where $\mathcal{I}$ is any one of the intervals $\mathcal{U}_{r}$ or $\mathcal{V}_{r}$, and $0 \leq j \leq J-1$. Summation over $r$ and $j$ will be postponed to the end of the proof. In order to interchange the order of summation here, we have to ascertain that $\left.H_{W, j}\right|_{W=\left(x^{m k}-n_{1}^{m k}\right)^{1 / m}}$ and $J$ do not 
depend on $n_{1} \in \mathcal{I}$. This is easy for $H$ : Call the corresponding values $H_{(\mathcal{I}, j)}$, and cf. formulae (6.8) and (6.13) below. It is a bit more delicate for $J$ : Let us observe that there exists a constant $b_{0}>1$ such that

$$
\frac{\max \left\{\left(x^{m k}-u^{m k}\right)^{1 /(m k)}: u \in \mathcal{I}\right\}}{\min \left\{\left(x^{m k}-u^{m k}\right)^{1 /(m k)}: u \in \mathcal{I}\right\}}<b_{0}
$$

for $\mathcal{I}$ any one of the intervals $\mathcal{U}_{r}$ or $\mathcal{V}_{r}$. Recalling (5.5) and the fact that now $W=\left(x^{m k}-n_{1}^{m k}\right)^{1 / m}$, we may choose, for every single $n_{1} \in \mathcal{I}$,

$$
c_{0}=\frac{\left(x^{m k}-n_{1}^{m k}\right)^{1 /(m k)}}{\min \left\{\left(x^{m k}-u^{m k}\right)^{1 /(m k)}: u \in \mathcal{I}\right\}} .
$$

Then $c_{0} \leq b_{0}$ throughout, and $J$ is the same for all $n_{1} \in \mathcal{I}$. Therefore,

$$
\begin{aligned}
& \left.\sum_{n_{1} \in \mathcal{I}} \sum_{H_{(\mathcal{I}, j)}, W, k, j}^{(\gamma)}\right|_{W=\left(x^{m k}-n_{1}^{m k}\right)^{1 / m}}= \\
& =\frac{e\left(-\frac{1}{8}\right)}{\sqrt{k-1}} \sum_{0<h<H_{(\mathcal{I}, j)}} \gamma_{h, H_{(\mathcal{I}, j)}} h \sum_{\ell=2^{j} h}^{2^{j+1} h}(h \ell)^{q / 2-1}\left(h^{q}+\ell^{q}\right)^{-1+1 /(2 q)} \times \\
& \times \sum_{n_{1} \in \mathcal{I}}\left(x^{m k}-n_{1}^{m k}\right)^{1 /(2 m k)} e\left(\left(x^{m k}-n_{1}^{m k}\right)^{1 /(m k)}\left(h^{q}+\ell^{q}\right)^{1 / q}\right) .
\end{aligned}
$$

Let $\Sigma(\mathcal{I})$ denote the complex conjugate of the innermost sum here, and put $N=$ $\left(h^{q}+\ell^{q}\right)^{1 / q} \asymp \ell$ for short. We transform this sum by Lemma 2, part (B), with $G(u)=\left(x^{m k}-u^{m k}\right)^{1 /(2 m k)}, F(u)=-N\left(x^{m k}-u^{m k}\right)^{1 /(m k)}$. Apart from the range of summation and the weight factors $G(n)$, this sum is quite similar to that in (5.6), with $(h, W, k)$ replaced by $\left(N, x^{m k}, m k\right)$. Thus Lemma $2,(\mathrm{~B})$, gives

$$
\begin{aligned}
\Sigma(\mathcal{I})=\frac{x N^{q_{1}-1 / 2}}{\sqrt{m k-1}} & \sum_{\ell_{1} \in F^{\prime}(\mathcal{I})} \ell_{1}^{q_{1} / 2-1}\left(N^{q_{1}}+\ell_{1}^{q_{1}}\right)^{1 / q_{1}-3 / 2} e\left(-x\left(N^{q_{1}}+\ell_{1}^{q_{1}}\right)^{1 / q_{1}}+\frac{1}{8}\right) \\
& + \text { error terms }
\end{aligned}
$$

with

$$
q_{1}:=\frac{m k}{m k-1}
$$

for short. To evaluate the error terms in (6.3), we have to distinguish if $\mathcal{I}$ stands for $\mathcal{U}_{r}$ or $\mathcal{V}_{r}$. Let us call the error terms arising finally in $(6.2) \delta\left(\mathcal{U}_{r}, j\right)$, resp., $\delta\left(\mathcal{V}_{r}, j\right)$. For $u \in \overline{\mathcal{U}}_{r}$, it follows that $G(u) \asymp x^{1 / 2}$, and $F^{\prime \prime}(u) \asymp \ell x^{1-m k}\left(2^{r} U\right)^{m k-2}$, hence

$$
\frac{G(u)}{\sqrt{\left|F^{\prime \prime}(u)\right|}} \asymp \ell^{-1 / 2} x^{m k / 2}\left(2^{r} U\right)^{1-m k / 2} \quad \text { for } u \in \overline{\mathcal{U}}_{r} .
$$

Thus the corresponding error term in (6.3) reads

$$
O\left(\ell^{-1 / 2} x^{m k / 2}\left(2^{r} U\right)^{1-m k / 2}\right)+O\left(x^{1 / 2} \log x\right) .
$$


Using this in (6.2) and summing over $\ell$ and $h$, we obtain

$$
\delta\left(\mathcal{U}_{r}, j\right)=O\left(x^{m k / 2}\left(2^{r} U\right)^{1-m k / 2} 2^{-j q / 2} \log x\right)+O\left(H_{\left(\mathcal{U}_{r}, j\right)}^{1 / 2} 2^{-j(q-1) / 2} x^{1 / 2} \log x\right),
$$

recalling from Lemma 1 that $\gamma_{h, H} h$ is bounded. Similarly, for $u \in \overline{\mathcal{V}}_{r}$, we have $G(u) \asymp\left(2^{-r}\right)^{1 /(2 m k)} x^{1 / 2}$, and $F^{\prime \prime}(u) \asymp \ell\left(2^{-r}\right)^{1 /(m k)-2} x^{-1}$, thus

$$
\frac{G(u)}{\sqrt{\left|F^{\prime \prime}(u)\right|}} \asymp 2^{-r} \ell^{-1 / 2} x \quad \text { for } u \in \overline{\mathcal{V}}_{r}
$$

Therefore, the error terms in (6.3) now read

$$
O\left(2^{-r} \ell^{-1 / 2} x\right)+O\left(\left(2^{-r}\right)^{1 /(2 m k)} x^{1 / 2} \log x\right)
$$

Using this in (6.2) and summing over $\ell$ and $h$, we arrive at

$$
\delta\left(\mathcal{V}_{r}, j\right)=O\left(2^{-r} 2^{-j q / 2} x \log x\right)+O\left(\left(2^{-r}\right)^{1 /(2 m k)} 2^{-j(q-1) / 2}\left(H_{\left(\mathcal{V}_{r}, j\right)} x\right)^{1 / 2} \log x\right)
$$

It remains to deal with the main term on the right hand side of $(6.3)$, let us call it $\Sigma^{*}(\mathcal{I})$

Case 1. $\mathcal{I}=\mathcal{U}_{r}$. For all $v \in F^{\prime}\left(\overline{\mathcal{U}}_{r}\right)$, we infer that $v \asymp \ell\left(2^{r} U\right)^{m k-1} x^{1-m k} \ll \ell$. Hence, putting

$$
\Sigma_{v}^{*}(\mathcal{I}):=\sum_{\ell_{1} \in F^{\prime}(\mathcal{I}), \ell_{1} \leq v} e\left(-x\left(N^{q_{1}}+\ell_{1}^{q_{1}}\right)^{1 / q_{1}}\right),
$$

summation by parts readily gives

$$
\Sigma^{*}\left(\mathcal{U}_{r}\right) \ll \ell^{1 / 2-q_{1} / 2} x\left(\ell\left(2^{r} U / x\right)^{m k-1}\right)^{q_{1} / 2-1} \max _{v \in \overline{\mathcal{U}}_{r}}\left|\Sigma_{v}^{*}\left(\mathcal{U}_{r}\right)\right|
$$

We shall estimate $\Sigma_{v}^{*}\left(\mathcal{U}_{r}\right)$ by van der Corput's "fourth derivative test": Writing $\phi(w)=$ $-x\left(N^{q_{1}}+w^{q_{1}}\right)^{1 / q_{1}}$, straightforward computation gives

$$
\begin{aligned}
\phi^{(4)}(w)= & -\left(q_{1}-1\right) x N^{q_{1}} w^{q_{1}-4}\left(N^{q_{1}}+w^{q_{1}}\right)^{1 / q_{1}-4} \times \\
& \times\left(N^{2 q_{1}}\left(2-q_{1}\right)\left(3-q_{1}\right)+(N w)^{q_{1}}\left(7-4 q_{1}\right)\left(1+q_{1}\right)+w^{2 q_{1}}\left(1+q_{1}\right)\left(2+q_{1}\right)\right) .
\end{aligned}
$$

For $1<q_{1} \leq \frac{7}{4}$, which is equivalent to $m k \geq \frac{7}{3}$, the last factor has all nonnegative coefficients. For $w \in F^{\prime}\left(\overline{\mathcal{U}}_{r}\right)$, it thus readily follows that $\phi^{(4)}(w) \asymp \ell^{-3} x\left(2^{r} U / x\right)^{4-3 m k}$. Further, as explained above, length $\left(F^{\prime}\left(\overline{\mathcal{U}}_{r}\right)\right) \ll \ell\left(2^{r} U / x\right)^{m k-1}$. In what follows, it will be convenient to set $2^{r} U / x=2^{-s}$, with $1 \leq s=R_{1}-r \leq R_{1}-1$. According to [13, p. 34, Theorem 2.6], uniformly in $v \in \overline{\mathcal{U}}_{r}$,

$$
\begin{aligned}
\Sigma_{v}^{*}\left(\mathcal{U}_{r}\right) \ll & \ell\left(2^{-s}\right)^{m k-1}\left(\ell^{-3} x\left(2^{-s}\right)^{4-3 m k}\right)^{1 / 14} \\
& +\left(\ell\left(2^{-s}\right)^{m k-1}\right)^{3 / 4}\left(\ell^{-3} x\left(2^{-s}\right)^{4-3 m k}\right)^{-1 / 14} \\
\ll & \ell^{11 / 14} x^{1 / 14}\left(2^{-s}\right)^{(11 m k-10) / 14}+\ell^{27 / 28} x^{-1 / 14}\left(2^{-s}\right)^{(27 m k-29) / 28}
\end{aligned}
$$


Using this in (6.6), we conclude that

$$
\Sigma^{*}\left(\mathcal{U}_{r}\right) \ll \ell^{2 / 7} x^{15 / 14}\left(2^{-s}\right)^{2(m k+1) / 7}+\ell^{13 / 28} x^{13 / 14}\left(2^{-s}\right)^{(13 m k-1) / 28} .
$$

We use this result in (6.2), and sum over $\ell$ and $h$, to arrive at

$$
\begin{aligned}
& \left.\sum_{n_{1} \in \mathcal{U}_{r}} \sum_{H_{\left(\mathcal{U}_{r}, j\right)}, W, k, j}^{(\gamma)}\right|_{W=\left(x^{m k}-n_{1}^{m k}\right)^{1 / m}} \\
& \ll\left(2^{j}\right)^{(4 k-11) /(14 k-14)} x^{15 / 14}\left(2^{-s}\right)^{2(m k+1) / 7} H_{\left(\mathcal{U}_{r}, j\right)}^{11 / 14} \\
& +\left(2^{j}\right)^{(13 k-27) /(28 k-28)} x^{13 / 14}\left(2^{-s}\right)^{(13 m k-1) / 28} H_{\left(\mathcal{U}_{r}, j\right)}^{27 / 28}+\delta\left(\mathcal{U}_{r}, j\right),
\end{aligned}
$$

where $\delta\left(\mathcal{U}_{r}, j\right)$ has been bounded in (6.4). We balance the first term at the right hand side against the error term $O\left(2^{-j q} 2^{-s} x^{2} H_{\left(\mathcal{U}_{r}, j\right)}^{-1}\right)$ which arises from the last sum in Theorem 2. (Recall that $\mathcal{U}_{r}$ is of length $\asymp 2^{r} U$ and $\left.] N_{j}, N_{j+1}\right]$ is of length $\asymp 2^{-j q} x$ : cf. (5.5). This gives

$$
H_{\left(\mathcal{U}_{r}, j\right)}=\left[\left(2^{-j}\right)^{(18 k-11) /(25 k-25)}\left(2^{-s}\right)^{(10-4 m k) / 25} x^{13 / 25}\right]+1
$$

Using this in (6.7) yields

$$
\begin{aligned}
& \left.\sum_{n_{1} \in \mathcal{U}_{r}} \sum_{H_{\left(\mathcal{U}_{r}, j\right)}, W, k, j}^{(\gamma)}\right|_{W=\left(x^{m k}-n_{1}^{m k}\right)^{1 / m}} \\
& \ll\left(2^{-j}\right)^{\frac{7 k+11}{25(k-1)}}\left(2^{-s}\right)^{\frac{4 m k+15}{25}} x^{37 / 25}+\left(2^{-j}\right)^{\frac{23 k+54}{100(k-1)}}\left(2^{-s}\right)^{\frac{31 m k+35}{100}} x^{143 / 100} \\
& +2^{-j q / 2}\left(2^{r} U\right)^{1-m k / 2} x^{m k / 2} \log x+\left(2^{-j}\right)^{\frac{9 k+7}{25(k-1)}}\left(2^{r} U / x\right)^{\frac{5-2 m k}{25}} x^{19 / 25} \log x .
\end{aligned}
$$

Summing finally over $j$ and $r$, resp., $s$, we arrive at ${ }^{(*)}$

$$
\begin{aligned}
& \sum_{U<n_{1} \leq \frac{1}{2} x} \Delta_{k}\left(\left(x^{m k}-\left|n_{1}\right|^{m k}\right)^{1 / m}\right) \\
& \ll x^{37 / 25}+U^{1-m k / 2} x^{m k / 2} \log x+U^{-(2 m k-5) / 25} x^{(2 m k+14) / 25} \log x .
\end{aligned}
$$

It remains to balance the middle term on the right hand side here against the error term $O\left(U x^{\frac{131}{208}}(\log x)^{\frac{18627}{8320}}\right)$ encountered at the beginning of this section. This leads to

$$
U \asymp x^{\frac{104 m k-131}{104 m k}}(\log x)^{-\frac{10307}{4160 m k}} .
$$

(*) Concerning the last term, we write up this conclusion for $5-2 m k<0$. In the contrary case, we obtain a term $x^{19 / 25+\varepsilon}$ instead which is negligible. 
With that choice of $U$, we finally infer from (6.9) that

$$
\begin{aligned}
& \sum_{\left|n_{1}\right| \leq \frac{1}{2} x} \Delta_{k}\left(\left(x^{m k}-\left|n_{1}\right|^{m k}\right)^{1 / m}\right) \\
& \ll x^{37 / 25}+x^{\frac{339 m k-262}{208 m k}}(\log x)^{\frac{18627 m k-20614}{8320 m k}}+x^{\frac{2238 m k-655}{2600 m k}+\varepsilon} \\
& \ll x^{37 / 25}+x^{\frac{339 m k-262}{208 m k}}(\log x)^{\frac{18627 m k-20614}{8320 m k}} .
\end{aligned}
$$

It remains to verify that $H_{\left(\mathcal{U}_{r}, j\right)}$, as defined by $(6.8)$, is $>1$ throughout. In fact, by $(5.5)$,

$$
H_{\left(\mathcal{U}_{r}, j\right)} \gg\left(2^{-J}\right)^{(18 k-11) /(25 k-25)} x^{13 / 25} \gg x^{\frac{13}{25}-0.53\left(\frac{18}{25}-\frac{11}{25 k}\right)}>x^{0.1384},
$$

if $m k \geq \frac{5}{2}$. But the case that $\frac{7}{3} \leq m k<\frac{5}{2}$ brings in only a factor

$$
\gg\left(2^{-s}\right)^{\frac{1}{25}\left(10-\frac{28}{3}\right)} \gg x^{-2 / 75} \text {. }
$$

Case 2. $\mathcal{I}=\mathcal{V}_{r}$. By a simple calculation, for all $v \in F^{\prime}\left(\overline{\mathcal{V}}_{r}\right)$, we have $v \asymp \ell 2^{r / q_{1}}$. Hence, with a look back to $(6.3)$, and $\Sigma_{v}^{*}\left(\mathcal{V}_{r}\right)$ as before, summation by parts yields

$$
\Sigma^{*}\left(\mathcal{V}_{r}\right) \ll 2^{-r} \ell^{-1 / 2} x \max _{v \in \overline{\mathcal{V}}_{r}}\left|\Sigma_{v}^{*}\left(\mathcal{V}_{r}\right)\right|
$$

We estimate again $\Sigma_{v}^{*}\left(\mathcal{V}_{r}\right)$ by the "fourth derivative test". Now

$$
\phi^{(4)}(w) \asymp \ell^{q_{1}} w^{-q_{1}-3} x \asymp 2^{-r(4-3 /(m k))} \ell^{-3} x
$$

for $w \in \overline{\mathcal{V}}_{r}$, hence, again by [13, p. 34, Theorem 6], uniformly in $v \in \overline{\mathcal{V}}_{r}$,

$$
\begin{aligned}
\Sigma_{v}^{*}\left(\mathcal{V}_{r}\right) \ll & 2^{r / q_{1}} \ell\left(2^{-r(4-3 /(m k))} \ell^{-3} x\right)^{1 / 14} \\
& +\left(2^{r / q_{1}} \ell\right)^{3 / 4}\left(2^{-r(4-3 /(m k))} \ell^{-3} x\right)^{-1 / 14} \\
\ll & 2^{r(10 m k-11) /(14 m k)} \ell^{11 / 14} x^{1 / 14}+2^{r(29 m k-27) /(28 m k)} \ell^{27 / 28} x^{-1 / 14} .
\end{aligned}
$$

Combining this with (6.11), we get

$$
\Sigma^{*}\left(\mathcal{V}_{r}\right) \ll 2^{-\frac{r(4 m k+11)}{14 m k}} \ell^{2 / 7} x^{15 / 14}+2^{\frac{r(m k-27)}{28 m k}} \ell^{13 / 28} x^{13 / 14} .
$$

Using this result in (6.2), and summing over $\ell$ and $h$, we obtain

$$
\begin{aligned}
& \left.\sum_{n_{1} \in \mathcal{V}_{r}} \sum_{H_{\left(\mathcal{V}_{r}, j\right)}, W, k, j}^{(\gamma)}\right|_{W=\left(x^{m k}-n_{1}^{m k}\right)^{1 / m}} \\
& \ll 2^{j \frac{4 k-11}{14(k-1)}} 2^{-r \frac{4 m k+11}{14 m k}} H_{\left(\mathcal{V}_{r}, j\right)}^{11 / 14} x^{15 / 14} \\
& +2^{j \frac{13 k-27}{28(k-1)}} 2^{r \frac{m k-27}{28 m k}} H_{\left(\mathcal{V}_{r}, j\right)}^{27 / 28} x^{13 / 14}+\delta\left(\mathcal{V}_{r}, j\right),
\end{aligned}
$$


where $\delta\left(\mathcal{V}_{r}, j\right)$ has been bounded in (6.5). The error term coming from the last sum in Theorem 2 now reads $O\left(2^{-j q} 2^{-r} x^{2} H_{\left(\mathcal{V}_{r}, j\right)}^{-1}\right)$, since $\mathcal{V}_{r}$ is of length $\asymp 2^{-r} x$. We balance this against the first term on the right hand side of (6.12) - except for the powers of $2^{r}$, with respect to which we simply compensate the factor $2^{r \frac{m k-27}{28 m k}}$ in the next-to-last term of (6.12). This gives

$$
H_{\left(\mathcal{V}_{r}, j\right)}=\left[2^{-j \frac{18 k-11}{25(k-1)}} 2^{-r \frac{m k-27}{27 m k}} x^{13 / 25}\right]+1
$$

With this choice of $H_{\left(\mathcal{V}_{r}, j\right)}$, we infer from (6.12) that

$$
\begin{aligned}
& \left.\sum_{n_{1} \in \mathcal{V}_{r}} \sum_{H_{\left(\mathcal{V}_{r}, j\right)}, W, k, j}^{(\gamma)}\right|_{W=\left(x^{m k}-n_{1}^{m k}\right)^{1 / m}}+O\left(2^{-j q} 2^{-r} x^{2} H_{\left(\mathcal{V}_{r}, j\right)}^{-1}\right) \\
& \ll\left(2^{-j}\right)^{\frac{7 k+11}{25(k-1)}}\left(\left(2^{-r}\right)^{\frac{26 m k+27}{27 m k}}+\left(2^{-r}\right)^{17 / 54}\right) x^{37 / 25}+\left(2^{-j}\right)^{\frac{23 k+54}{100(k-1)}} x^{143 / 100} \\
& +2^{-j q / 2} 2^{-r} x \log x+\left(2^{-j}\right)^{\frac{9 k+7}{25(k-1)}} 2^{-r / 54} x^{19 / 25} \log x .
\end{aligned}
$$

Obviously $H_{\left(\mathcal{V}_{r}, j\right)}>1$ throughout: As before after (6.10),

$$
H_{\left(\mathcal{V}_{r}, j\right)} \gg x^{0.1384} 2^{-r \frac{m k-27}{27 m k}} \gg x^{0.1384-1 / 27} \gg x^{1 / 10}
$$

Since on the right hand side of (6.14) all exponents of 2 are negative, summation over $r$ and $j$ yields

$$
\sum_{\frac{1}{2} x<\left|n_{1}\right| \leq x} \Delta_{k}\left(\left(x^{m k}-\left|n_{1}\right|^{m k}\right)^{1 / m}\right)=O\left(x^{37 / 25}\right) .
$$

Together with (6.10) and (4.7), this completes the proof of Theorem 1.

\section{References}

[1] Chamizo F, Cristóbal E, Ubis A (2009) Lattice points in rational ellipsoids. J. Math. Anal. Appl. 350: $283-289$

[2] Copson ET (1965) Asymptotic expansions. Cambridge: Univ. Press

[3] Graham SW, Kolesnik G (1991) Van der Corput's method of exponential sums. Cambridge: Univ. Press

[4] Haberland K (1993) Über die Anzahl der Gitterpunkte in konvexen Gebieten. Preprint FSU Jena (unpublished)

[5] Heath-Brown DR (1999) Lattice points in the sphere. In: Györy et al. (eds.) Number theory in progress, vol. 2, 883-892. Berlin: de Gruyter

[6] Hlawka E (1954) Über Integrale auf konvexen Körpern I. Monatsh Math 54: 1-36, II, ibid. 54: 81-99

[7] Huxley MN (1996) Area, lattice points, and exponential sums. LMS Monographs, New Ser. 13, Oxford: University Press

[8] Huxley MN (2003) Exponential sums and lattice points III. Proc. London Math. Soc. (3) 87: 591-609 
[9] Iosevich A, Sawyer E, Seeger A (2002) Mean square discrepancy bounds for the number of lattice points in large convex bodies. J. Anal. Math. 87: 209-230

[10] Ivić A (1996) The Laplace transform of the square in the circle and divisor problems. Stud. Sci. Math. Hung. 32: 181-205

[11] Ivić A, Krätzel E, Kühleitner M, Nowak WG (2006) Lattice points in large regions and related arithmetic functions: Recent developments in a very classic topic. In: Proceedings Conf. on Elementary and Analytic Number Theory ELAZ'04, held in Mainz, May 24-28, W. Schwarz and J. Steuding eds., Franz Steiner Verlag, pp. 89-128.

[12] Iwaniec H, Kowalski E (2004) Analytic Number Theory, AMS Coll.Publ. 53. Providence, R.I.

[13] Krätzel E (1988) Lattice points. Berlin: VEB Deutscher Verlag der Wissenschaften

[14] Krätzel E (1999) Lattice points in super spheres. Comment. Math. Univ. Carolinae 40: $373-391$

[15] Krätzel E (2000) Analytische Funktionen in der Zahlentheorie. Wiesbaden: Teubner.

[16] Krätzel E (2000) Lattice points in three-dimensional large convex bodies. Math. Nachr. 212: $77-90$

[17] Krätzel E (2002) Lattice points in three-dimensional convex bodies with points of Gaussian curvature zero at the boundary. Monatsh. Math. 137: 197-211

[18] Krätzel E (2002) Lattice points in some special three-dimensional convex bodies with points of Gaussian curvature zero at the boundary. Comment. Math. Univ. Carolinae 43: 755-771

[19] Krätzel E, Nowak WG (2007) Eine explizite Abschätzung für die Gitter-Diskrepanz von Rotationsellipsoiden. Monatsh. Math 152: 45-61

[20] Krätzel E, Nowak WG (2008) The lattice discrepancy of bodies bounded by a rotating Lamé's curve. Monatsh. Math 154: 145-156

[21] Kuba G (1993) On sums of two $k$-th powers of numbers in residue classes II. Abh. Math. Sem. Hamburg 63: $87-95$

[22] Kühleitner M, Nowak WG (2000) The asymptotic behavior of the mean-square of fractional part sums. Proc. Edinburgh Math. Soc. 43: 309-323

[23] Müller W (1999) Lattice points in large convex bodies. Monatsh. Math. 128: 315-330

[24] Nowak WG (1986) On the lattice rest of a convex body in $\mathbb{R}^{s}$, II. Arch. Math. (Basel) 47: 232-237

[25] Nowak WG (2008) On the lattice discrepancy of bodies of rotation with boundary points of curvature zero. Arch. Math. (Basel) 90: 181-192

[26] Peter M (2002) Lattice points in convex bodies with planar points on the boundary. Monatsh. Math. 135: $37-57$

[27] Vaaler JD (1985) Some extremal problems in Fourier analysis. Bull. Amer. Math. Soc. 12: $183-216$

\author{
Ekkehard Krätzel \\ Faculty of Mathematics \\ University of Vienna \\ Nordbergstraße 15 \\ 1090 Wien, Österreich \\ Werner Georg Nowak \\ Institute of Mathematics \\ Department of Integrative Biology \\ Universität für Bodenkultur Wien \\ Gregor Mendel-Straße 33 \\ 1180 Wien, Österreich \\ E-mail: nowak@boku.ac.at
}

